\title{
Mapping the Electronic Surface Potential of Nanostructured Surfaces
}

\author{
P. Ruffieux, ${ }^{1, *}$ K. Aït-Mansour, ${ }^{1}$ A. Bendounan, ${ }^{2}$ R. Fasel, ${ }^{1}$ L. Patthey,${ }^{3}$ P. Gröning,,${ }^{1}$ and O. Gröning ${ }^{1}$ \\ ${ }^{1}$ Empa, Swiss Federal Laboratories for Materials Testing and Research, Feuerwerkerstrasse 39, 3602 Thun, Switzerland \\ ${ }^{2}$ Synchrotron Soleil, L'Orme des Merisiers, Saint-Aubin, B.P. 48, F-91192 Gif-sur-Yvette Cedex, France \\ ${ }^{3}$ Swiss Light Source, Paul Scherrer Institut, CH-5232 Villigen PSI, Switzerland
}

(Received 22 August 2008; published 27 February 2009)

\begin{abstract}
We present a method for the quantitative determination of the surface potential landscape of nanostructured surfaces based on the local analysis of the lowest field emission resonances by scanning tunneling spectroscopy. The method has a lateral resolution of $\sim 1 \mathrm{~nm}$ and is applied to elucidate the sitespecific adsorption properties of the strain relief pattern formed by two monolayers of $\mathrm{Ag}$ on $\mathrm{Pt}(111)$. For the example of $\mathrm{C}_{60}$ fullerenes, we show that the surface potential difference of up to $0.35 \mathrm{eV}$ is responsible for the site-selective immobilization on the strain relief pattern.
\end{abstract}

DOI: 10.1103/PhysRevLett.102.086807

A detailed knowledge of the local electronic properties of solids and their surfaces is crucial for an understanding of a large variety of processes, ranging from electron scattering in transport phenomena to catalytic reactions, which show a pronounced site specificity. Recently, sitespecific adsorption on inorganic surfaces has been reported for organic molecules on laterally inhomogeneous surfaces consisting mainly of varying stacking sequences of the atomic layers [1-3]. However, the understanding of the relevant local physical properties responsible for the sitespecific substrate-molecule interactions remains very poor.

Among the first important parameters investigated for surfaces is the work function, which defines the minimum energy required for removing an electron from a metal to infinity at $0 \mathrm{~K}$. However, a number of important surfacerelated phenomena, such as catalytic processes [4] and electron emission [5], cannot be described with this macroscopic work function but require knowledge of local variations of the electrostatic potential close to the surface. Recently, it has been shown that lateral dipoles related to variations of the local work function might influence the interaction of polarizable adsorbates with the substrate [6], demonstrating the need for a method allowing a quantitative determination of the local surface potential landscape. The first experimental work enabling the measurement of the local work function was based on photoemission of adsorbed xenon (PAX), which probes the surface potential at about $0.2 \mathrm{~nm}$ in front of the surface via the Xe electron states, which are pinned to the local vacuum level due to a weak coupling of the Xe atoms to the surface [7]. Kelvin probe force microscopy [8] is capable of detecting variations of the local contact potential with a spatial resolution of several tens of nanometers. A higher resolution is achieved by measuring the apparent barrier height with scanning tunneling microscopy (STM) [9], but the assumptions required for extracting the local work function severely limit its precision. An alternative way is the analysis of field emission resonances (FERs), which are detected with STM when applying voltages larger than the tip work
PACS numbers: 73.30.+y, 68.37.Ef, 68.43.Hn, 73.20. $-\mathrm{r}$

function. Their sensitivity to variations of the surface potential has first been discussed by Binnig et al. [10] and has been applied for the qualitative description of work function modulations of thin ionic [11] and oxide [12] films grown on metal surfaces. Recently, Ploigt et al. [13] combined the experimental investigation of FERs with calculations of the eigenstates in the potential well formed between tip and sample for the determination of the work function differences between the first few $\mathrm{NaCl}$ layers and the bare underlying substrate.

Here we report on a method for the determination of the surface potential landscape with a resolution of $\sim 1 \mathrm{~nm}$, which is based on the combination of an energy level mapping of the lowest FERs and their simulation using a 1D model potential between STM tip and surface. The method is applied for explaining site-specific interactions of $\mathrm{C}_{60}$ molecules deposited on a nanostructured template surface formed by the deposition of two monolayers (MLs) of silver on $\mathrm{Pt}(111)$.

Experiments have been performed with a lowtemperature STM (Omicron) working under ultrahigh vacuum conditions (base pressure $2 \times 10^{-10} \mathrm{mbar}$ ). The $\mathrm{Ag} / \mathrm{Pt}$ strain relief pattern has been prepared as described in Ref. [14]. Spectroscopic $z(V)$ curves were recorded at $5 \mathrm{~K}$ with a closed feedback loop (current set point $I=$ $50 \mathrm{pA}$ ) and differentiated numerically. PAX has been performed at the X09LA-SIS beam line of the Swiss Light Source by dosing Xe onto the strain relief pattern at $56 \mathrm{~K}$. Spectra have been recorded at $35 \mathrm{~K}$ with a photon energy of $84 \mathrm{eV}$ and an overall resolution of $50 \mathrm{meV}$.

Deposition and subsequent annealing $(800 \mathrm{~K})$ of two MLs of silver on $\operatorname{Pt}(111)$ leads to a periodic strain relief pattern exhibiting a high degree of long-range order. According the atomic model proposed by Brune et al. [15], strain built up by the $4.3 \% \mathrm{Ag} / \mathrm{Pt}$ lattice mismatch is relieved by the formation of face-centered cubic (fcc) and hexagonal close-packed (hcp) stacking regions separated by partial dislocations along [110] located at the interface region [16] and appearing as depressions in the 
STM image (Fig. 1). In analogy to this model, we call fcc the quasihexagonal domain and $\mathrm{hcp}_{1}$ and $\mathrm{hcp}_{2}$ the smaller and larger triangular domains, respectively, contained in the unit cell of the $\mathrm{Ag} / \mathrm{Pt}$ strain relief pattern, which can be regarded as a $(25 \times 25)$ superstructure with respect to $\mathrm{Pt}(111)$.

Regarding molecular adsorption on the $\mathrm{Ag} / \mathrm{Pt}$ strain relief pattern, we find a highly inhomogeneous immobilization on the different surface domains. In order to determine the energetically favorable adsorption sites of $\mathrm{C}_{60}$, molecules have been deposited at low coverage $(\sim 0.05 \mathrm{ML})$ and low temperature $(\sim 150 \mathrm{~K})$ followed by annealing to room temperature (RT). In contrast to slow deposition at RT [14], we find selective adsorption of individual molecules and small clusters ( $\leq 3$ molecules) in the $\mathrm{hcp}_{1}$ region. The annealing step leads preferentially to the formation of larger close-packed molecular clusters in the $\mathrm{hcp}_{1}$ region [Fig. 1(c)], which is related to the high cohesive energy of $\mathrm{C}_{60}$ [3] and a reduced mobility of molecular clusters compared to individual molecules. This brings up the question of which local physical property determines the site-selective immobilization of $\mathrm{C}_{60}$. We will show below that the hcp ${ }_{1}$ region is favored due to a low local surface potential.

The lateral resolution of this method is determined by measuring the spatial variation of the FERs in the vicinity of a point defect consisting of a single adsorbed $\mathrm{C}_{60}$ molecule, which locally modifies the surface dipole. Figure 2 displays $d z / d V$ spectra acquired along the indicated line. The energy positions of the FERs are clearly shifted for spectra recorded over the molecule. The lateral extent over which the energy position varies sensitively increases with the order of the FER. The main contribution to the decreasing sharpness is given by the increasing separation between the surface and higher order FER, leading to an effective Coulomb potential that is smeared out for a surface potential that varies within a few tenths of nanometers. For comparison, we plot the spatial expecta-

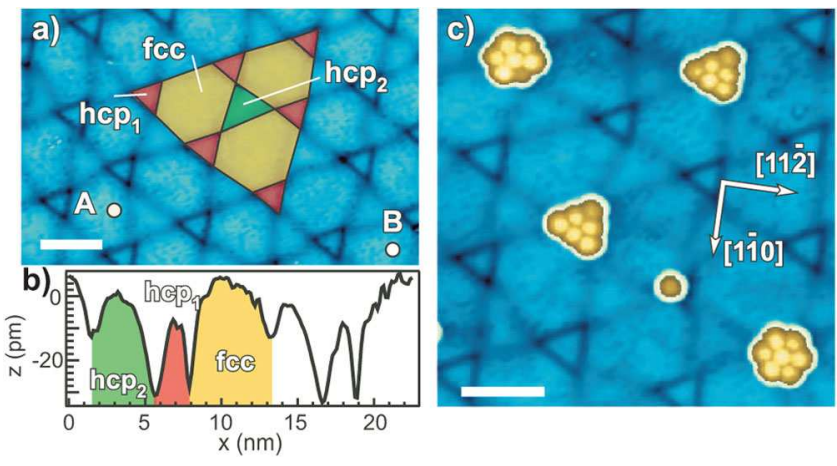

FIG. 1 (color online). STM images of the $2 \mathrm{ML} \mathrm{Ag/Pt(111)}$ strain relief pattern. (a) Topography image revealing the three domains of the pattern $\left(T=77 \mathrm{~K}, V_{s}=-2 \mathrm{~V}, I=2 \mathrm{nA}\right)$. (b) Height profile from $A$ to $B$, as shown in (a). (c) Topography image of $\mathrm{C}_{60}$ adsorbed on the pattern $\left(V_{s}=-2 \mathrm{~V}\right.$, $I=0.1 \mathrm{nA})$. Scale bars: $5 \mathrm{~nm}$. tion value of the hydrogenic image states, given by $\langle z\rangle=$ $6 a_{0} n^{2}$, where $a_{0}$ is the Bohr radius [17]. In Fig. 2, we show the values of $\langle z\rangle$ for the first four FERs centered on the left edge of the $\mathrm{C}_{60}$ molecule, which qualitatively explains the lateral range over which the level energy changes. The experimental resolution is systematically better than the calculated $\langle z\rangle$ values. This can be understood by considering the increased constriction of the FERs to the surface due to the applied positive sample bias [12]. Accordingly, the spatial expectation values of the image states, i.e., the resonances without applied field, give an upper limit for the estimation of the lateral experimental resolution, yielding $\sim 1 \mathrm{~nm}$ for the surface potential determination based on the analysis of the two lowest FERs.

In order to model the FER for a varying surface potential, we use a similar approach as recently presented by Ploigt et al. [13] by numerically solving the onedimensional nonrelativistic Schrödinger equation in the direction perpendicular to the surface. The electric potential $V(z)$ between the tip and the sample is calculated by taking into account the image potential of the tip, the tipsample distance $d$, and the applied sample bias $V_{s}$ assuming a planar tip geometry [Fig. 3(b)]. For the image potential at the surface, we have taken into account the image plane located at $z_{i}$ leading to the expression

$$
V(z)=\frac{-1}{4 \pi \varepsilon_{0}} \frac{e^{2}}{4}\left(\frac{1}{z}+\frac{1}{z-\left(z_{t}-z_{i}\right)}\right)-\frac{V_{s}-\Delta \varphi / e}{z_{t}} \cdot z
$$

with the distance between the tip and the sample $z_{t}=$ $z_{v}\left(V_{s}\right)+z_{0}$, where $z_{v}\left(V_{s}\right)$ is the experimental tip retraction

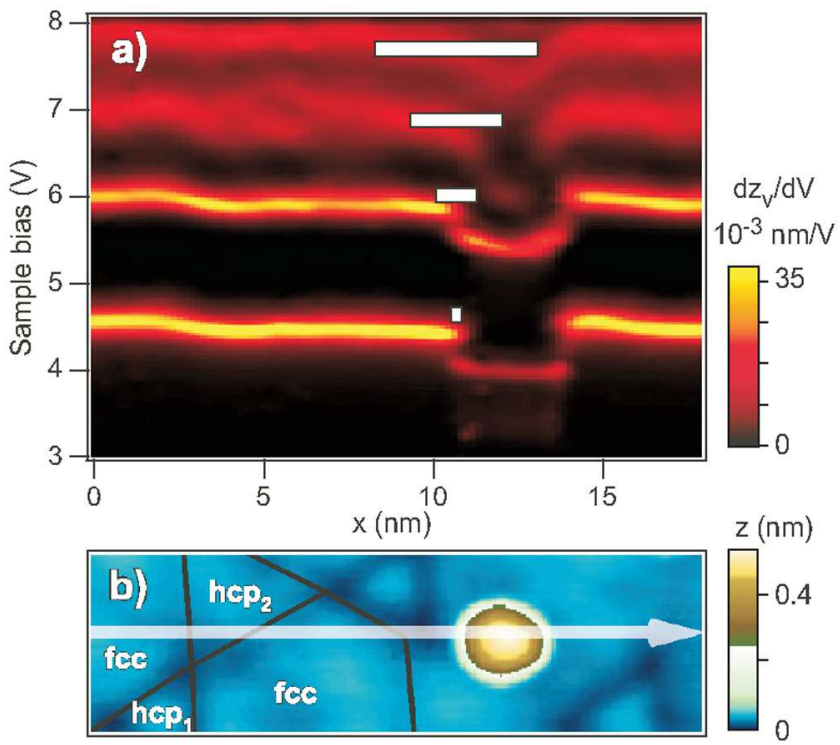

FIG. 2 (color online). (a) Series of $100 d z / d V$ spectra $(\Delta x=$ $0.18 \mathrm{~nm}$ ) recorded along the line indicated in (b) and represented as a color-coded image. The white bars represent the spatial expectation values of the first four FERs (see text). (b) Topography image of a single $\mathrm{C}_{60}$ adsorbed at a defect in the fcc region $\left(V_{s}=-2 \mathrm{~V}, I=0.1 \mathrm{nA}\right)$. 


\section{(a)}
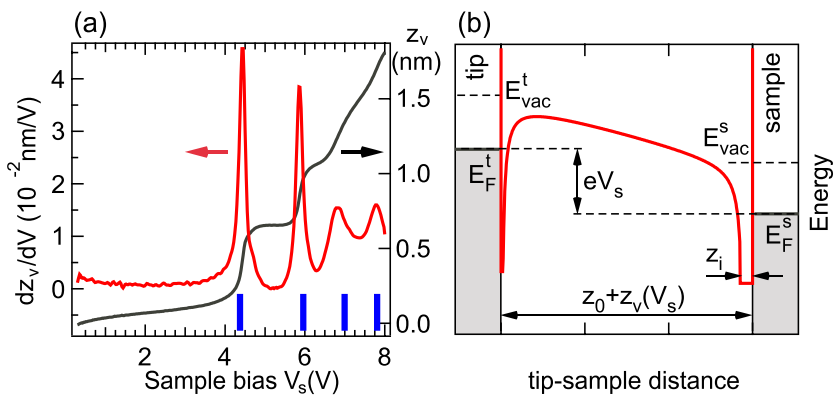

FIG. 3 (color online).

(a) Experimental $z_{v}\left(V_{s}\right)$ curve (black line) and $d z_{v} / d V\left(V_{s}\right)$ curve (red line) recorded in the center of the hcp ${ }_{2}$ domain. (b) Relevant parameters of the model potential used for the simulation of the FER (see text). The unknown parameters are fitted to reproduce the energy position of the FER in the $\mathrm{hcp}_{2}$ region, revealing $\phi_{t}=4.0 \mathrm{eV}, z_{i}=0.17 \mathrm{~nm}$, and $z_{0}=1.7 \mathrm{~nm}$. The related energy levels of the modeled FER are shown as vertical bars for $n=1, \ldots, 4$ in (a).

curve and $z_{0}$ is the tip-sample distance at the onset of the spectroscopy curve. The potential between the image plane $z_{i}$ and the surface is set to a constant value of $E_{F}-10 \mathrm{eV}$ corresponding to the minimum potential in the bulk of silver [13]. The weak coupling between the FER and the bulk states is simulated by setting the potential inside the tip and the sample to large positive values. The unknown parameters, i.e., tip work function $\phi_{t}$ and image plane positions $z_{i}$ and $z_{0}$, are determined by fitting the energy position of the simulated FERs to the four measured ones in the $\mathrm{hcp}_{2}$ region where the smallest lateral variation of the energy position of the FERs is observed. These parameters are then kept constant, and only the local work function $\Delta \varphi$ is varied in the model potential in order to find the best agreement with the measured FERs.

Figure 4 shows the analysis of a series of spectra taken along a line crossing the different domains of the strain relief pattern. For the first FER, we see a peak shift of 0.23 (1) V when comparing spectra recorded in the fcc and the $\mathrm{hcp}_{1}$ domain. In order to get the highest possible lateral resolution for the surface potential mapping, only the first two FERs are taken into account for the analysis. The according local work function shift resulting from our analysis is of the order of $0.35 \mathrm{eV}$ with the minimum located in the hcp ${ }_{1}$ region and the maximum in the center of the fcc region [Fig. 4(c)]. The local work function of the $\mathrm{hcp}_{2}$ region is only slightly higher $[+0.06(1) \mathrm{eV}]$ than in the hcp $\mathrm{p}_{1}$ region.

A similar analysis has been performed for a set of spectra acquired on a dense two-dimensional (2D) grid in the vicinity of the hcp ${ }_{1}$ region allowing the determination of the 2D surface potential landscape [Fig. 5(a)]. This analysis reveals a broad minimum of the local work function in the $\mathrm{hcp}_{1}$ region and a maximum slope along the [112] direction near the border between $\mathrm{hcp}_{1}$ and fcc domains. The related maximum electric field amounts to $0.23(2) \mathrm{V} / \mathrm{nm}$ in this direction. We have performed a similar analysis where only the first FER $(\langle z\rangle=0.3 \mathrm{~nm})$

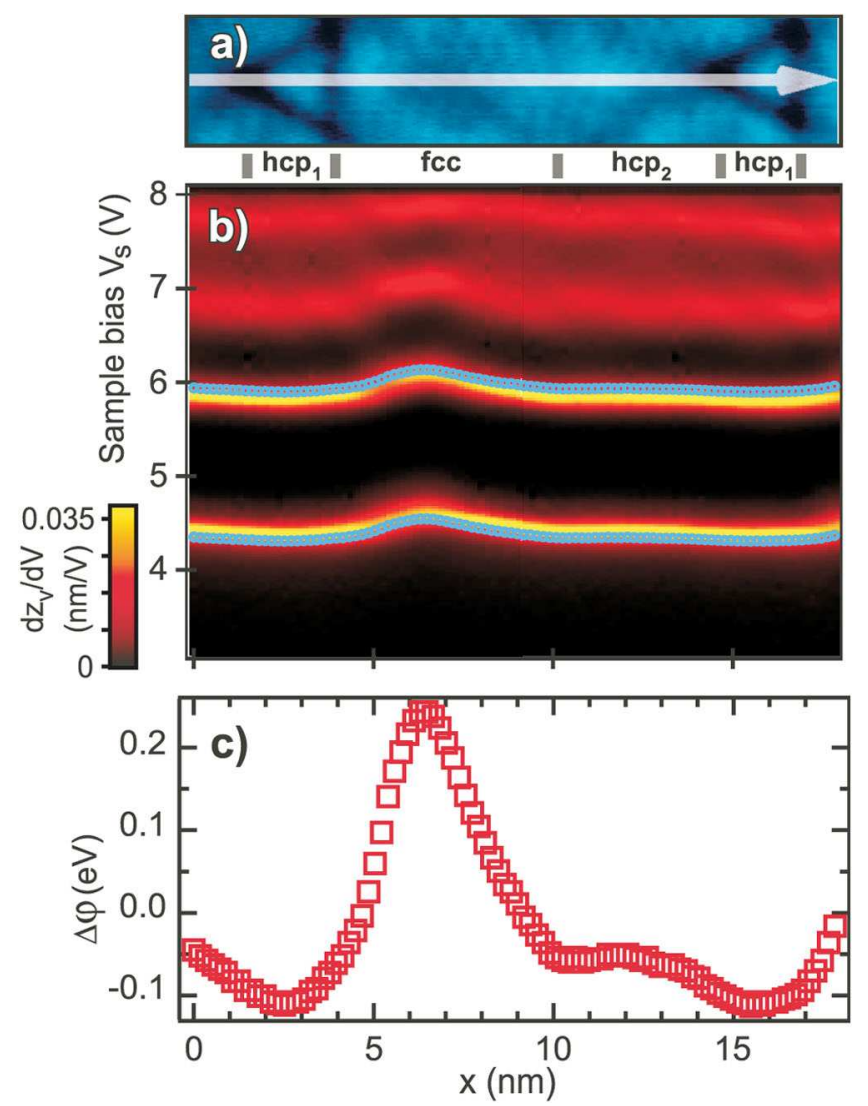

FIG. 4 (color online). (a) Topography image acquired simultaneously with spectroscopic data $\left(V_{s}=0.5 \mathrm{~V}\right)$. (b) Color-coded representation of a series of $100 d z / d V(V)$ spectra recorded along the line indicated in (a). (c) Variation of the local surface potential as determined with a fit of $\Delta \varphi$ in the model potential considering the two lowest FERs. Fitted energy positions are overlaid as open circles to the experimental data in (b).

has been included in the fitting procedure. No significant differences in the resulting slopes of the potential landscape can be detected, indicating that they are not limited by the resolution of our method.

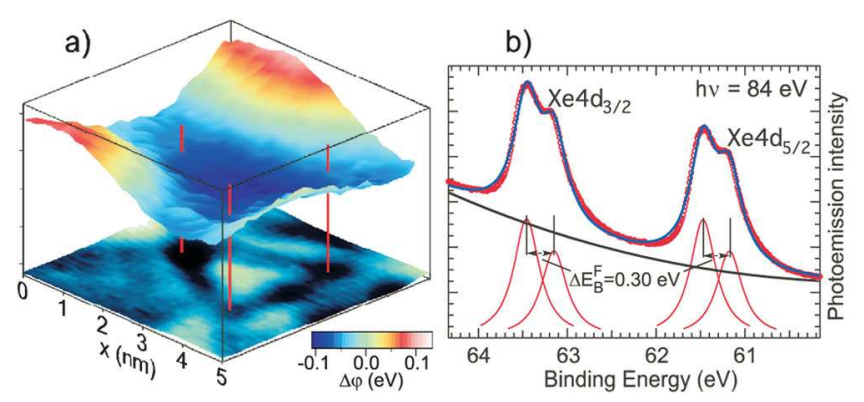

FIG. 5 (color online). (a) Three-dimensional representation of the surface potential landscape near the $\mathrm{hcp}_{1}$ region as determined by applying the fitting procedure to the $30 \times 30$ spectra acquired on the $5 \times 5 \mathrm{~nm}^{2}$ scan area. The bottom image shows the simultaneously acquired topography image. (b) Photoelectron spectrum of the $\mathrm{Xe} 4 \mathrm{~d}$ states recorded from the $\mathrm{Ag} / \mathrm{Pt}(111)$ strain relief pattern covered with $0.7(2) \mathrm{ML}$ of $\mathrm{Xe}$. 
In order to further corroborate the appropriateness of the proposed method for a quantitative determination of the surface potential landscape, we have performed PAX experiments. This method, beside two-photon photoemission spectroscopy, is well established as a local work function probe for heterogeneous surfaces with the limitation of the missing simultaneous imaging of the nanostructures [4]. Figure 5(b) shows a Xe4d PAX spectrum for a 0.7(2) ML Xe coverage. In contrast to PAX spectra recorded on $\mathrm{Ag}(111)$, on the $\mathrm{Ag} / \mathrm{Pt}$ strain relief pattern both spin-orbit split states reveal a broad distribution characterized by two main contributions (FWHM $0.30 \mathrm{eV}$ ) separated by $0.30 \mathrm{eV}$. Since the binding energies of the states $E_{B}^{F}$ (with respect to $\left.E_{F}\right)$ are directly linked to local variations of the work function by $\Delta E_{B}^{F}(i, j) \cong-\Delta \varphi(i, j)$ at two dissimilar surface sites $i$ and $j$ [4], this indicates a broad distribution of the local work function within the unit cell with two main contributions separated by $0.30 \mathrm{eV}$. In order to compare these results to the FER-based values, we have performed a histogram analysis of the local surface potential across the unit cell of the strain relief pattern. This analysis yields a broad distribution with two dominant contributions centered at -0.08 (mainly hcp ${ }_{1}$ and $h \mathrm{hp}_{2}$ ) and $0.2 \mathrm{eV}$ (fcc). Their separation of $0.28 \mathrm{eV}$ is in excellent agreement with PAX revealing an underestimation of the surface potential variation by $\sim 7 \%$ for the FER-related results when compared to the PAX results.

In the case of the $\mathrm{Ag} / \mathrm{Pt}(111)$ strain relief pattern, the variation of the local surface potential is firmly related to local changes of the in-plane lattice parameter $a$. From a careful analysis of atomic resolution STM images (not shown), we find average lattice parameters of 299(5), 289(4), and 280(4) pm for hcp , hcp $_{2}$, and fcc domains, respectively. From an electron (and hence dipole) density point of view, this directly suggests a lowering of the work function for increasing $a$. This is in good agreement with recent density function theory calculations [18], where an increase of the lattice parameter of a $\operatorname{Ag}(111)$ slab by $20 \mathrm{pm}$ yielded a work function reduction of $0.3 \mathrm{eV}$.

Regarding the selective adsorption of $\mathrm{C}_{60}$ in the hcp region, the presented mapping of the local work function allows us to draw some important conclusions. An increased binding energy due to induced dipoles in the molecule can be excluded since in this case molecules are expected to have an affinity to the largest surface dipoles, i.e., to regions with the highest work function [19]. Similarly, dipoles related to lateral electric fields imposed by local variations of the surface potential are not the relevant contribution, since the maximum lateral electric field is located in the fcc regions. Although effects related to a varying local density of states (LDOS) in the topmost Ag layer cannot be excluded, they are expected to play a minor role since no significant shift of the molecular orbitals is observed when comparing molecules adsorbed in the center of the $\mathrm{hcp}_{1}$ region to molecules adsorbed on

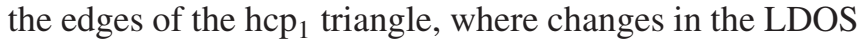
are most pronounced (not shown). The most prominent variation is found for the local surface potential, which is expected to sensitively influence the molecule-substrate interaction if the interaction has at least partially ionic character. For $\mathrm{C}_{60}$ adsorption on noble metals this is indeed the case: Besides the main covalent part, also ionic contributions are involved in the interaction with $\mathrm{Ag}(111)$ [20], making it sensitive to variations of the local work function.

In conclusion, we have presented a method for a quantitative 2D mapping of the surface potential with a lateral resolution of $\sim 1 \mathrm{~nm}$. For the $\mathrm{Ag} / \mathrm{Pt}$ strain relief pattern, we find a variation of the local work function of $0.35 \mathrm{eV}$, which is attributed to a locally varying lattice parameter. The selective immobilization of $\mathrm{C}_{60}$ on the $\mathrm{hcp}_{1}$ domain is attributed to the low local work function, which favors electron transfer to the molecule and thus increases the interaction to the substrate due to the partially ionic nature of the interaction.

*pascal.ruffieux@empa.ch

[1] M. Vladimirova et al., Europhys. Lett. 56, 254 (2001).

[2] J. Barth, Annu. Rev. Phys. Chem. 58, 375 (2007).

[3] W. Xiao et al., J. Phys. Chem. B 110, 21394 (2006).

[4] K. Wandelt, Appl. Surf. Sci. 111, 1 (1997).

[5] G. Barnes, Phys. Rev. 97, 1579 (1955).

[6] H. Dil et al., Science 319, 1824 (2008).

[7] K. Wandelt, Thin Metal Films and Gas Chemisorption (Elsevier, Amsterdam, 1987).

[8] J. M. R. Weaver and D. W. Abraham, J. Vac. Sci. Technol. B 9, 1559 (1991).

[9] Y. Hasegawa, J. F. Jia, K. Inoue, A. Sakai, and T. Sakurai, Surf. Sci. 386, 328 (1997).

[10] G. Binnig et al., Phys. Rev. Lett. 55, 991 (1985).

[11] M. Pivetta, F. Pathey, M. Stengel, A. Baldereschi, and W.-D. Schneider, Phys. Rev. B 72, 115404 (2005).

[12] E. D. L. Rienks, N. Nilius, H.-P. Rust, and H.-J. Freund, Phys. Rev. B 71, 241404 (2005).

[13] H.-C. Ploigt, C. Brun, M. Pivetta, F. Patthey, and W.-D. Schneider, Phys. Rev. B 76, 195404 (2007).

[14] K. Aït-Mansour, P. Ruffieux, W. Xiao, P. Gröning, R. Fasel, and O. Gröning, Phys. Rev. B 74, 195418 (2006).

[15] H. Brune, H. Röder, C. Boragno, and K. Kern, Phys. Rev. B 49, 2997 (1994).

[16] K. Ait-Mansour et al., Nano Lett. 8, 2035 (2008).

[17] C. Wong et al., J. Phys. Chem. B 103, 282 (1999).

[18] M. J. Verstraete et al., Phys. Rev. B 70, 205427 (2004).

[19] N. Nilius, E. D. L. Rienks, H.-P. Rust, and H.-J. Freund, Phys. Rev. Lett. 95, 066101 (2005).

[20] L.-L. Wang and H.-P. Cheng, Phys. Rev. B 69, 165417 (2004). 